\title{
Current epidemiology and antenatal presentation of Posterior Urethral Valves: Outcome of BAPS CASS national study
}

\author{
Ewan Brownlee ${ }^{1}$, Ruth Wragg ${ }^{1}$, Andy Robb ${ }^{1}$, Marian Knight ${ }^{2}$, Liam McCarthy ${ }^{1}$ \\ ${ }^{1}$ Department of Paediatric Urology, Birmingham Children's Hospital, UK \\ ${ }^{2}$ National Perinatal Epidemiology Unit, Oxford University, UK
}

\section{Aim of the study}

Posterior urethral valves (PUV) are commonest cause of congenital bladder outlet obstruction (BOO) in boys, and of ESRF in childhood. In the 1990s, 1 in 4,000 boys had PUV: $1 / 3$ presented antenatally, $1 / 3$ postnatally in infancy, $1 / 3$ late ( $>1$ year). This study aimed to ascertain the proportion presenting antenatally, postnatally and late in a contemporary cohort and to identify any differences between these patient groups.

\section{Methods}

A national audit (BAPS CASS) of all referrals in the UK of boys diagnosed with suspected or confirmed PUV in a year was conducted. National registration data provided male birthrate.

Data presented as number (\%), analysed by Mann-Whitney U-test and Chi-square test, with $p<0.05$ taken as significant.

Ethics approval: NRES Committee South Central Oxford A (12/SC/0416)

\section{Main Results}

Data collected October 2014 - September 2015, 24/25 centres submitted data (96\%). Male birth rate: 432,806/yr. 121 cases BOO; PUV 113 (93\%). Incidence of BOO 1/3,580; PUV $1 / 3,800$ per annum. PUV boys presented: antenatally $40(35 \%)$; in infancy $47(42 \%)$; late 26(23\%).

Plasma creatinine higher in antenatally diagnosed BOO vs. postnatal, 54(39.5-109.5) $\mu \mathrm{mols} / \mathrm{l}$ vs. 34(21-47) $\mu \mathrm{mols} / \mathrm{l}, \mathrm{P}=0.0005$. Hydronephrosis and ureteric dilatation significantly greater in antenatally diagnosed BOO vs postnatal vs late. Renal dysplasia (cortical thinning, poor corticomedullary differentiation or renal cysts) significantly more likely in antenatally diagnosed BOO.

\section{Conclusion}

Incidence of PUV appears not to have changed in 30 years ( 1/4000), nor the proportion antenatally diagnosed ( $1 / 3)$. Boys antenatally diagnosed have significantly higher postnatal plasma creatinine, more hydro-ureteronephrosis and renal dysplasia than those diagnosed in infancy or late. It may be hypothesized that this is the reason they are detected antenatally. 
Keywords: posterior urethral valves; epidemiology; incidence; antenatal. Level of evidence: Ilb - National cohort study 


\section{Introduction}

Posterior urethral valves (PUV) are the commonest cause of congenital bladder outlet obstruction (BOO) and only occur in boys. A quarter of patients with posterior urethral valves develop end stage renal failure (ESRF), and this is the commonest cause of ESRF in childhood (1). 30 years ago, Parkhouse et al (2) reported equal proportions of patients presenting antenatally, postnatally in infancy and late (>1year). Since that time, antenatal screening has increased and ultrasound technology has improved, so we aimed to ascertain whether the timing of presentation for patients with posterior urethral valves has changed and whether there are any differences between patients diagnosed antenatally and those presenting postnatally or late.

We aimed to describe for the first time the epidemiology of a national cohort of all patients presenting with posterior urethral valves (PUV) and congenital bladder outlet obstruction (BOO) and to ascertain whether there is any correlation between timing of presentation and severity of associated renal disease.

\section{Methods}

A national cohort was identified of all patients referred to participating paediatric surgical units in the UK and Ireland with suspected or confirmed posterior urethral valves from October 2014 to October 2015. The British Association of Paediatric Surgeons Congenital Anomaly Surveillance System (BAPS-CASS) was used, as has been previously described (Gastroschisis paper - ref). Each month, routine reporting cards were sent to nominated reporting clinicians in all paediatric surgical units in the UK and Ireland, requesting details of the number of patients presenting to their unit that month with a new diagnosis of bladder outlet obstruction, i.e. suspected or confirmed posterior urethral valves. In response to a monthly card return indicating that there had been a case of PUV, clinicians were sent a form requesting further details including antenatal course, diagnosis, associated anomalies, renal function, ultrasound results, management and other outcomes for the first 3 months after initial diagnosis. Up to three reminders were sent if the first form was not returned. Duplicate cases were identified and removed and participating clinicians were contacted by email or telephone to seek any missing data. National vital registration data was used to provide the total and male birthrate during the same period (England and Wales - Office for National Statistics: ons.gov.uk; Scotland - National Records of Scotland: nrscotland.gov.uk; Ireland - Central Statistics Office: cso.ie).

Plasma creatinine, as measured at 1 month post-treatment, was used as a marker of renal function. Hydronephrosis was quantified by the Renal Pelvic Antero-Posterior Diameter (APD)) and presence of ureteric dilatation was recorded. Renal dysplasia was assessed by a combination of cortical thinning, poor corticomedullary differentiation or renal cysts on ultrasound scan.

Data were collated and analysed; results are expressed as medians (interquartile ranges), rates with $95 \%$ confidence intervals $(\mathrm{Cl})$ or numbers and percentages as appropriate. Groups 
were compared using the Mann-Whitney U-test or Chi-square test as appropriate, with $\mathrm{p}<0.05$ taken as significant.

Ethical approval was sought and granted: NRES Committee South Central Oxford A (12/SC/0416)

\section{Results}

Twenty-five out of 26 (96\%) centres submitted patient data (the centre that did not contribute was the quaternary referral centre for the UK).

One hundred and twenty-one patients were identified with a new diagnosis of BOO between October 2014 and September 2015 inclusive. Of these, a complete dataset was available for 120 (99\%). 113 (93\%) had a confirmed diagnosis of PUV. Table 1 describes the timing and underlying diagnosis of $\mathrm{BOO}$; other causes of $\mathrm{BOO}$ included 4 complete membranes (urethral atresia), 3 Cobbs collars, and 1 anterior urethral syringocele. This gives an incidence of $4 / 432,806$ or $1 / 100,000$ boys with urethral atresia; for Cobb's collar the incidence was $3 / 432,806$, or $1 / 133,000$ live born-boys; and for anterior syringocele $1 / 432,806$, or $\sim 1 / 400,000$ boys.

The number of live male births for this year in the UK and Ireland was 432,806 , thus the incidence of BOO was 1 in 3,580 (95\% Cl 1 in 3,000 to 1 in 4,310) and the incidence of PUV was 1 in 3,800 (95\% Cl 1 in 3,190 to 1 in 4,650). In the same year, the over-all number of live births was 843,259, so the incidence of BOO and PUV was 1 in 6,970 (95\% Cl 1 in 5,830 to 1 in 8,400$)$ and 1 in 7,460 (95\% Cl 1 in 6,210 to 1 in 9,060) live births respectively.

The timing of diagnosis for BOO patients was antenatally in 45 patients (37\%), 48 (40\%) were diagnosed postnatally within the first year of life, and 28 (23\%) were diagnosed late (beyond 12 months of age). Thirty-five percent of boys with PUV were diagnosed antenatally $(n=40), 42 \%$ in infancy $(n=47)$, and $23 \%$ late $(n=26)$ (see Table 1$)$.

Those presenting postnatally were all diagnosed on the basis of micturating cystourethrogram (MCUG); indications for MCUG were: investigation of antenatal hydronephrosis, urinary tract infections, incontinence and renal impairment (see Table 2).

96 (79\%) patients had isolated PUV and 25 (21\%) had at least one other associated anomaly. Median gestational age at birth was 39 weeks (IQR 37-40 weeks) and median birth weight was $3.21 \mathrm{~kg}$ (IQR $2.88-3.74 \mathrm{~kg}$ ). Only one patient (0.8\%) had a family history of PUV.

Only one patient died in this series (pulmonary hypoplasia associated with PUV). The mortality rate for $\mathrm{BOO}$ was therefore $0.8 \%(95 \% \mathrm{Cl} 0.02-4.5 \%)$.

Plasma creatinine was significantly higher in boys with antenatally diagnosed BOO vs.

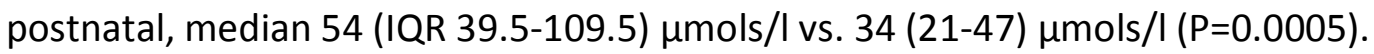
Hydronephrosis and ureteric dilatation were significantly greater in antenatally diagnosed $\mathrm{BOO}$ vs postnatal vs late (table 3 ). Renal dysplasia was significantly more likely in antenatally diagnosed $\mathrm{BOO}$ (see table 3). 


\section{Discussion}

This national cohort study provides a contemporary and comprehensive picture of the incidence and epidemiology of PUV in the UK. The current incidence of PUV in the UK is 1 in 3,800 live-born boys, and is very similar to previous reports $(1 / 4,000)(2)$. Antenatal presentation of PUV accounted for $35 \%$, postnatal presentation in the first year accounted for $42 \%$, presentation after 1 year $23 \%$. This is the first study to show that renal function, hydroureteronephrosis and renal dysplasia vary according to presentation, being most abnormal with antenatal presentation.

There appears to have been no significant change in incidence of PUV in 30 years. This study looked at all boys in the UK regardless of age at presentation, in a similar way to the work of Parkhouse et al. Other studies have confirmed a similar incidence of close to 1 in 8,000 births or 1 in 4,000 boys - Atwell et al in the UK in the 1980s and more recently (2004-2009) Thakkar et al in New South Wales, Australia $(1 / 7,800)(3)(4)$. These studies all included postnatal and late diagnosed PUV. The incidence of BOO diagnosed antenatally has been reported as $1 / 3,000$, with $69 \%$ being due to PUV, giving an incidence of $1 / 4,400$ (5). This report did not include any postnatal or late diagnosed cases so represents an underestimate for PUV boys. Incidences of 1/25,000 are historical and do not represent modern rates for PUV.

The incidence of rare causes of congenital bladder outlet obstruction is poorly described. This is the first report to allow the incidence of these rare cause of BOO to be calculated. No incidence is available for urethral atresia (6). Four boys with urethral atresia survived to birth, gives an incidence of $1 / 100,000$ boys, noting that this will be a low estimate as there is considerable hidden antenatal mortality for this condition. Cobbs collar is a bulbar urethral lesion in an infra-sphincteric position, that is associated with a variable degree of obstruction, (7)(8)(9). The incidence of this condition is not well described. In this national cohort, the incidence was $\sim 1 / 133,000$ live born-boys. Similarly anterior syringocele is a rare entity due to cystic dilatation of Cowper's, with no published incidence in boys (10). In this national cohort, anterior syringocele occurred in 1/400,000 boys.

The mortality rate for congenital $\mathrm{BOO}$ and PUV has fallen progressively over the last 50 years. In the 1960 s this was reported by DI Williams as $25 \%$, predominantly due to the combination of uraemia and urosepsis in infancy (11). In the 1970 s to early 1980 s it was reported by Atwell as 7\% (3) and now this study suggests it is less than $1 \%$. Antenatal diagnosis became widespread in the 1980s, so some patients (a third) could be detected before developing urosepsis (2).

This study is the first study to show a statistically significant relationship between timing of diagnosis and increased risk of renal impairment. Other studies have attempted to correlate timing of diagnosis of PUV with severity of disease. Hutton et al (12) identified worse outcomes in boys with an antenatal diagnosis before 24 weeks gestation. Kibar et al (13) 
compared outcomes of patients diagnosed before and after 1 year of life: although the results did not reach statistical significance due to small sample size in that publication, those in the early diagnosis group had higher rates of renal insufficiency (48\% vs $25 \%$ ) and need for transplantation ( $10 \%$ vs $0 \%$ ). Plasma creatinine was significantly higher in antenatally presenting $\mathrm{BOO}$ and PUV, as were hydroureteronephrosis and renal dysplasia.

Why, despite increased antenatal screening and improved ultrasound technology, are PUV boys not being increasingly recognised antenatally over the past 30 years, as was predicted in the 1980s (14)? This is the first study that correlates the renal ultrasound appearances in PUV boys and mode of presentation: the most dysplastic and dilated kidneys are those that are detectable and therefore recognised antenatally. The median anterior to posterior diameter (APD) of the renal pelvis of the antenatal group was $9.5(0-19) \mathrm{mm}$, whereas in the postnatal group the median APD was only $5.5(0-10) \mathrm{mm}$, which would be regarded by most centres as within normal limits, indeed would be below the criteria for upper tract dilatation (UTD) 1 (15). Those presenting over one year of age had a still smaller median APD of 0(09) $\mathrm{mm}$ (see Table 3). Correlating with timing of presentation is the appearance of the kidneys, with renal dysplasia (as assessed by a combination of cortical thinning, poor corticomedullary differentiation or renal cysts) significantly more common in patients diagnosed antenatally compared to those diagnosed postnatally and those diagnosed beyond one year old with significantly lower rates of renal abnormalities on ultrasound (15).

Although the rate of antenatal diagnosis remains the same as 30 years ago, a slightly higher proportion of those postnatally diagnosed are identified in the first year of life. This may relate to increased investigation of patients with urinary tract infections.

A limitation of this study is that plasma creatinine decreases in the first 3-6 months of age as renal maturation occurs. This could potentially explain the apparently better renal function in the later presenting patients. However, creatinine improves with bladder drainage in those presenting antenatally, and it might have been expected that those 'missed' cases of PUV which present late would have worsened renal function due to prolonged inadequate drainage. However, this was not found in this national cohort. That the renal dysplasia and hydroureteronephrosis is significantly worse in the antenatal compared to the postnatal and late presentations strongly supports a genuine difference in renal function between these groups.

$25 / 26$ (96\%) UK paediatric surgical centres submitted data to this national audit. The one centre which did not participate is a quaternary referral centre and it is therefore possible that any patients seen there would also overlap with other centres' data collection. This study therefore makes a powerful observation of the current incidence of this congenital condition.

The incidence of PUV does not appear to have signifcantly changed in the past 30 years and neither has the rate of antenatal diagnosis. This study is the first to show that patients presenting antenatally have statistically significantly worse renal function, increased upper tract dilatation and more dysplastic parenchyma. This, at least in part, explains why the rate of antenatal diagnosis has not increased: patients 'missed' antenatally are milder cases with 
less or no hydronephrosis and significantly less dysplasia. In the 1980s it was expected that the majority of congenital uropathies including PUV would be detectable antenatally, but this has not been achieved, and may not be achievable (14). Antenatal diagnosis has however allowed the detection of the most severely affected boys and is likely to be associated with the $95 \%$ reduction of infant mortality in this condition.

\section{Conclusion}

The incidence of PUV appears not to have changed in 30 years (remaining approximately 1 in 4000) and nor has the proportion of patients antenatally diagnosed (which is around 1 in 3). Boys antenatally diagnosed have a significantly higher postnatal plasma creatinine, more hydro-ureteronephrosis and renal dysplasia than those diagnosed in infancy or late. It may be hypothesised that this is the reason they are detected antenatally.

\section{Funding}

This paper presents independent research arising from a Research Professorship award to Prof Marian Knight, NIHR-RP-011-032, supported by the National Institute for Health Research. The views expressed in this publication are those of the author(s) and not necessarily those of the NHS, the National Institute for Health Research or the Department of Health.

\section{References}

1. Woolf AS, Thiruchelvam N. Congenital Obstructive Uropathy: Its Origin and Contribution to End-Stage Renal Disease in Children. Advances in Renal Replacement Therapy. 2001 Jul 1;8(3):157-63.

2. Parkhouse HF, Barratt TM, Dillon MJ, Duffy PG, Fay J, Ransley PG, et al. Long-term Outcome of Boys with Posterior Urethral Valves. British Journal of Urology. 62(1):59-62.

3. Atwell JD. Posterior urethral valves in the British isles:A multicenter B.A.P.S. review. Journal of Pediatric Surgery. 1983 Feb 1;18(1):70-4.

4. Thakkar D, Deshpande AV, Kennedy SE. Epidemiology and demography of recently diagnosed cases of posterior urethral valves. Pediatr Res. 2014 Dec;76(6):560-3.

5. Malin G, Tonks AM, Morris RK, Gardosi J, Kilby MD. Congenital lower urinary tract obstruction: a population-based epidemiological study. BJOG: An International Journal of Obstetrics \& Gynaecology. 2012 Nov 1;119(12):1455-64.

6. RESERVED IU--AR. Orphanet: Atresia of urethra [Internet]. [cited 2018 May 27]. Available from: https://www.orpha.net/consor/cgibin/OC_Exp.php?lng=EN\&Expert $=105$ 
7. Cobb BG, Wolf JA, Ansell JS. Congenital stricture of the proximal urethral bulb. J Urol. 1968 May;99(5):629-31.

8. Dewan PA, Keenan RJ, Morris LL, Le Quesne GW. Congenital urethral obstruction: Cobb's collar or prolapsed congenital obstructive posterior urethral membrane (COPUM). Br J Urol. 1994 Jan;73(1):91-5.

9. Dewan PA, Goh DG. Variable expression of the congenital obstructive posterior urethral membrane. Urology. 1995 Mar;45(3):507-9.

10. Maizels M, Stephens FD, King LR, Firlit CF. Cowper's syringocele: a classification of dilatations of Cowper's gland duct based upon clinical characteristics of 8 boys. J Urol. 1983 Jan;129(1):111-4.

11. Williams DI, Whitaker RH, Barratt TM, Keeton JE. Urethral Valves1. British Journal of Urology. 45(2):200-10.

12. Hutton KAR, Thomas DFM, Arthur RJ, Irving HC, Smith SEW. Prenatally Detected Posterior Urethral Valves: Is Gestational age at Detection a Predictor of Outcome? The Journal of Urology. 1994 Aug 1;152(2, Part 2):698-701.

13. Kibar Y, Ashley RA, Roth CC, Frimberger D, Kropp BP. Timing of posterior urethral valve diagnosis and its impact on clinical outcome. J Pediatr Urol. 2011 Oct;7(5):538-42.

14. Thomas DF, Gordon AC. Management of prenatally diagnosed uropathies. Arch Dis Child. 1989 Jan;64(1 Spec No):58-63.

15. Nguyen HT, Benson CB, Bromley B, Campbell JB, Chow J, Coleman B, et al. Multidisciplinary consensus on the classification of prenatal and postnatal urinary tract dilation (UTD classification system). J Pediatr Urol. 2014 Dec;10(6):982-98.

\section{Legends:}

Table 1. Diagnoses by Timing of Presentation

Table 2. Indications for MCUG in Patients Presenting Postnatally (patients in the $>1$ year group often had more than one indication for MCUG)

Table 3. Severity of Renal Disease by Timing of Presentation (*median (IQR)) 


\begin{tabular}{|l|c|c|c|}
\hline \multicolumn{1}{|c|}{ Diagnosis } & Antenatal & Postnatal (infancy) & Late (>1 yr) \\
\hline BOO (121) & $45(37 \%)$ & $48(40 \%)$ & $28(23 \%)$ \\
$\begin{array}{r}\text { True PUV (113) } \\
\text { Other BOO } \\
\text { Complete Membrane/Urethral Atresia } \\
\text { Cobb's Collar }\end{array}$ & $40(35 \%)$ & $47(42 \%)$ & $26(23 \%)$ \\
Anterior Syringocele & 3 & 1 & 2 \\
\hline
\end{tabular}




\begin{tabular}{|l|r|r|}
\hline \multirow{2}{*}{ Indication for MCUG } & \multicolumn{2}{|c|}{ Number of patients } \\
\cline { 2 - 3 } & $<1$ year & $>1$ year \\
\hline Antenatal hydronephrosis & 27 & \\
UTI & 38 & 11 \\
Renal impairment & & 3 \\
Incontinence & & 16 \\
Other & & 16 \\
\hline
\end{tabular}




\begin{tabular}{|c|c|c|c|c|}
\hline Findings at Diagnosis & Antenatal & Postnatal (infancy) & Late (>1 yr) & P Value \\
\hline Creatinine (micromol/I)* & $54(39.5-109.5)$ & \multicolumn{2}{|c|}{$34(21-47)$} & 0 \\
\hline Renal units & 81 & 54 & 40 & \\
\hline$A P D(m m)^{*}$ & $9.5(0-19)$ & $5.5(0-10)$ & $0(0-9)$ & 0 \\
\hline Hydroureter $(\mathrm{mm})^{*}$ & $5(0-8)$ & $0(0-7)$ & $0(0)$ & 0 \\
\hline Renal dysplasia & 46 (56\%) & $30(41 \%)$ & $8(20 \%)$ & 0 \\
\hline Cortical Thinning & $29(35 \%)$ & $21(28 \%)$ & $4(10 \%)$ & 0 \\
\hline Poor CMD & $31(38 \%)$ & $19(26 \%)$ & $4(10 \%)$ & 0 \\
\hline Cortical Cysts & $20(24 \%)$ & 14 (19\%) & $0(0 \%)$ & 0 \\
\hline
\end{tabular}

